\title{
ON THE DECOMPOSITION OF INFINITELY DIVISIBLE CHARACTERISTIC FUNCTIONS WITH CONTINUOUS POISSON SPECTRUM
}

\author{
ROGER CUPPENS ${ }^{1}$
}

1. Introduction. Let $f$ be an infinitely divisible characteristic function of the real variable $t$. It is well known (see, for example, [3, Theorem 5.5.2]) that $f$ admits the so-called Lévy's representation

$$
\begin{aligned}
\log f(t)= & i t a-\frac{1}{2} \sigma^{2} t^{2}+\int_{-\infty}^{0}\left(e^{i t u}-1-i t u\left(1+u^{2}\right)^{-1}\right) d M(u) \\
& +\int_{0}^{\infty}\left(e^{i t u}-1-i t u\left(1+u^{2}\right)^{-1}\right) d N(u),
\end{aligned}
$$

where $M, N, a$ and $\sigma$ satisfy the following conditions:

(a) $a$ is a real constant;

(b) $\sigma^{2}$ is a nonnegative constant;

(c) $M$ and $N$ are nondecreasing functions in the intervals $(-\infty, 0)$ and $(0,+\infty)$ respectively;

(d) $M(-\infty)=N(+\infty)=0$;

(e) $\int_{-\epsilon}^{0} u^{2} d M(u)+\int_{0}^{e} u^{2} d N(u)<+\infty$ for any $\epsilon>0$.

In the following, we denote the set of all the infinitely divisible characteristic functions without indecomposable factors by $I_{0}$ and $S(\mu)$ means the set of the points of increase (or spectrum) of the function $\mu$.

In the case when at least one of the spectra $S(M)$ and $S(N)$ of the functions $M$ and $N$ in the representation (1) contains an interval, the following results are known.

TheOREM A (CRAmÉr [1]). If there exist two positive constants $k$ and $c$ such that at least one of the relations $M^{\prime}(u)>k$ almost everywhere in $(-c, 0)$ or $N^{\prime}(u)>k$ almost everywhere in $(0, c)$ holds, then the function $f$ defined by (1) does not belong to $I_{0}$.

TheOREM B (Shimizu [5]). If there exist three positive constants $k, c$ and $d(d>2 c)$ such that at least one of the relations $M^{\prime}(u)>k$ almost everywhere in $(-d,-c)$ or $N^{\prime}(u)>k$ almost everywhere in $(c, d)$ holds, then the function $f$ defined by (1) does not belong to $I_{0}$.

\footnotetext{
Received by the editors March 25, 1968.

${ }^{1}$ This work was supported by the National Science Foundation Grant NSF GP6175.
} 
THEOREM C (Ostrovskiİ [4]). If the following conditions are satisfied:

(a) $\sigma=0$;

(b) $S(M)=\varnothing$;

(c) there exists a positive constant $c$ such that $S(N) \subset[c, 2 c]$, then the function $f$ defined by (1) belongs to $I_{0}$.

In the present note, we prove, using a modification of the method of Cramér [1], a result which supplements the preceding theorems and we give some remarks on the structure of $I_{0}$.

\section{The main result.}

THEOREM 1. Let $f$ be the characteristic function defined by

$$
\log f(t)=\int_{-\infty}^{+\infty}\left(e^{i t u}-1-i t u\left(1+u^{2}\right)^{-1}\right) \alpha(u) d u,
$$

where

$$
\begin{aligned}
& \alpha(u)=k \quad \text { if }-c\left(1+2^{-n}\right)<u<-c \text { or } d<u<d\left(1+2^{-n}\right), \\
& =0 \text { otherwise, }
\end{aligned}
$$

$k, c$ and $d$ being positive constants and $n$ being a positive integer. Then $f$ has an indecomposable factor.

Proof. We give the demonstration in the particular case $c=d=1$. The proof in the general case follows the same lines, but is more intricate.

Let $\beta$ be the function

$$
\begin{aligned}
\beta(u) & =k & & \text { if } 1<|u|<1+2^{-n} \\
& =-k \epsilon & & \text { if }|u|<2^{-n} \\
& =0 & & \text { otherwise. }
\end{aligned}
$$

If we prove that the function $g$ defined by

$$
\log g(t)=\int_{-\infty}^{+\infty}\left(e^{i t u}-1-i t u\left(1+u^{2}\right)^{-1}\right) \beta(u) d u
$$

is a characteristic function for some $\epsilon>0$, then clearly $g$ divides $f$ and, since $g$ is not infinitely divisible, the theorem will be proved (cf. [3, Theorem 6.2.2]).

Let $\alpha_{m}$ and $\beta_{m}$ be the functions defined by

$$
\alpha_{1}(x)=\alpha(x) ; \quad \alpha_{m}(x)=\int_{-\infty}^{+\infty} \alpha_{m-1}(x-t) \alpha_{1}(t) d t ;
$$




$$
\beta_{1}(x)=\beta(x) ; \quad \beta_{m}(x)=\int_{-\infty}^{+\infty} \beta_{m-1}(x-t) \beta_{1}(t) d t .
$$

We denote by $K(m)$ the constant $K(m)=m\left(1+2^{-n}\right)$ and prove by induction that

$$
\alpha_{m}(x)=\beta_{m}(x) \quad \text { if }|x|>K(m)-1 .
$$

This is true for $m=1$. Suppose that

$$
\alpha_{m-1}(x)=\beta_{m-1}(x) \quad \text { if }|x|>K(m-1)-1 .
$$

From the properties of convolutions, we have

$$
\alpha_{m}(x)=\beta_{m}(x)=0 \quad \text { if }|x| \geqq K(m) .
$$

Moreover, if $K(m)-1<x<K(m)$, then

$$
\begin{aligned}
\alpha_{m}(x) & =\int_{-\infty}^{+\infty} \alpha_{m-1}(x-t) \alpha_{1}(t) d t=k \int_{-K(1)}^{\rho} \alpha_{m-1}(x-t) d t \\
& =k \int_{-K(1)}^{\rho} \beta_{m-1}(x-t) d t=\beta_{m}(x)
\end{aligned}
$$

where $\rho=\inf (-1, K(m-1)-x)$. The case $-K(m)<x<-K(m)+1$ being exactly the same, (3) is proved. Therefore, for any $m$,

$$
\beta_{m}(x) \geqq 0 \quad \text { if }|x| \geqq K(m)-1 .
$$

Now, we prove that

$$
\lim _{\epsilon \rightarrow 0} \sup _{|x| \leqq K(m)-1}\left|\alpha_{m}(x)-\beta_{m}(x)\right|=0 .
$$

In fact, if $\epsilon<1$, we have

$$
\left|\alpha_{m}(x)\right|<[K(2)]^{m-1} k^{m}, \quad\left|\beta_{m}(x)\right|<[K(2)]^{m-1} k^{m},
$$

and from these formulae and from

$$
\begin{aligned}
\alpha_{m}(x)-\beta_{m}(x)= & \int_{-\infty}^{+\infty} \alpha_{m-1}(x-t) \alpha_{1}(t) d t-\int_{-\infty}^{+\infty} \beta_{m-1}(x-t) \beta_{1}(t) d t \\
= & \int_{-\infty}^{+\infty} \alpha_{m-1}(x-t)\left[\alpha_{1}(t)-\beta_{1}(t)\right] d t \\
& -\int_{-\infty}^{+\infty}\left[\beta_{m-1}(x-t)-\alpha_{m-1}(x-t)\right] \beta_{1}(t) d t
\end{aligned}
$$

it follows by induction that 


$$
\left|\alpha_{m}(x)-\beta_{m}(x)\right|<\epsilon(2 k)^{m}[K(2)]^{m-1}
$$

and (5) is proved.

The next step is the proof of the formula

$$
S\left(\alpha_{m}\right)=\bigcup_{j=0}^{m}[j-K(m-j), K(j)+j-m] .
$$

This is true for $m=1$. Now $S\left(\alpha_{m+1}\right)=S\left(\alpha_{m}\right)(+) A$, where

$$
A=[-K(1),-1] \cup[1, K(1)] \text {, }
$$

and $(+)$ indicates the vectorial sum. Therefore

$$
\begin{aligned}
S\left(\alpha_{m+1}\right) & =\bigcup_{j=0}^{m}[j-K(m-j)-K(1), K(j)+j-m-1] \\
& \cup \bigcup_{j=0}^{m}[j-K(m-j)+1, K(j)+j-m+K(1)] \\
& =\bigcup_{j=0}^{m}[j-K(m+1-j), K(j)+j-m-1] \\
& \cup \bigcup_{j=1}^{m+1}[j-K(m+1-j), K(j)+j-m-1]
\end{aligned}
$$

and (6) is proved.

(6) implies that $S\left(\alpha_{m}\right)$ is all the interval $[-K(m), K(m)]$ if $m \geqq c(1)$ where $c(j)$ means the constant $2^{n+1}+j$. But, from the definition of $\alpha_{m}$ as convolution of nonnegative functions, we have

$$
\inf _{|x|<K(c(j))-1} \alpha_{c(j)}(x)>0 \quad j=2, \cdots, c(3)
$$

and

$$
\inf _{|x|<K(c(2))-1}\left[\sum_{j=1}^{c(2)} \frac{1}{j !} \alpha_{j}(x)\right]>0 .
$$

Therefore, from (5), if $\epsilon$ is small enough,

$$
\inf _{|x|<K(c(j))-1} \beta_{c(j)}(x)>0, \quad j=2, \cdots, c(3)
$$

and

$$
\inf _{|x|<K(c(2))-1}\left[\sum_{j=1}^{e(2)} \frac{1}{j !} \beta_{j}(x)\right]>0 .
$$

From the definition of $\beta_{m}$, we have for $k<m$ 


$$
\beta_{m}(x)=\int_{-\infty}^{\infty} \beta_{m-k}(x-t) \beta_{k}(t) d t
$$

and from (4), (7) and the properties of convolutions it follows that if $\epsilon$ is small enough

$$
\beta_{m}(x) \geqq 0, \quad m>c(2)
$$

and this, with (8), implies that

$$
\sum_{j=1}^{\infty} \frac{1}{j !} \beta_{j}(x)>0
$$

for any $x$ if $\epsilon$ is small enough. Letting

$$
G(x)=e^{-\lambda}\left\{\chi(x+\eta)+\int_{-\infty}^{x}\left[\sum_{j=1}^{\infty} \frac{1}{j !} \beta_{j}(y+\eta)\right] d y\right\}
$$

where $\chi(x)$ is the degenerate distribution and

$$
\begin{aligned}
& \lambda=\int_{-\infty}^{+\infty} \beta(x) d x, \\
& \eta=\int_{-\infty}^{+\infty} x\left(1+x^{2}\right)^{-1} \beta(x) d x,
\end{aligned}
$$

then $G$ is, from (9), a distribution function if $\epsilon$ is small enough and it is easily shown that the belonging characteristic function is $g$. That completes the proof of the theorem.

From this theorem and from Theorems A and B of the introduction, we deduce easily the

COROLLARY. Let $f$ be an infinitely divisible characteristic function and suppose that the functions $M$ and $N$ which occur in its Lévy's representation satisfy the following condition: There exist five constants $a, b, c, d$ and $k(0 \leqq a<b, 0 \leqq c<d, k>0)$ such that $M^{\prime}(u)>k$ almost everywhere in $(-b,-a)$ and $N^{\prime}(u)>k$ almost everywhere in $(c, d)$. Then $f$ has an indecomposable factor.

3. Remarks. Let $f$ be the infinitely divisible characteristic function defined by (1). If we define the functions $f_{N}, f_{+}$and $f_{-}$by

$$
\begin{aligned}
& \log f_{N}(t)=i t a-\frac{1}{2} \sigma^{2} t^{2} \\
& \log f_{+}(t)=\int_{0}^{+\infty}\left(e^{i t u}-1-i t u\left(1+u^{2}\right)^{-1}\right) d N(u), \\
& \log f_{-}(t)=\int_{-\infty}^{0}\left(e^{i t u}-1-i t u\left(1+u^{2}\right)^{-1}\right) d M(u),
\end{aligned}
$$


we have a natural decomposition of $f, f=f_{N} f_{+} f_{-}$, as product of three infinitely divisible characteristic functions.

It is well known that we can have the following circumstance: $f_{+}$ belongs to $I_{0}, f_{-}=1$ while $f$ does not belong to $I_{0}$ (clearly $f_{N}$ belongs to $\left.I_{0}\right)$. Such examples can be constructed, for example, from the Theorem 8.1.1 of Linnik [2].

From the particular case of the preceding corollary when $b \leqq 2 a$, $d \leqq 2 c$ and from the theorem of Ostrovskir stated in the introduction, we have examples of another circumstance: $f_{+}$and $f_{-}$belong to $I_{0}$ while, for $f_{N}=1, f$ does not belong to $I_{0}$.

Such examples with discontinuous Poisson spectra are given by the

THEOREM 2. Let $f$ be the infinitely divisible characteristic function defined by

$$
\begin{aligned}
\log f(t)= & \lambda[\exp (i p t)+\exp (-i p t)-2] \\
& +\mu[\exp (i(p+2) t)+\exp (-i(p+2) t)-2]
\end{aligned}
$$

where $p$ is an integer greater than two and $\lambda$ and $\mu$ are positive constants. Then $f$ has an indecomposable factor.

Proof. The method is a modification of one of P. Lévy (see [3, pp. 178-179]). We distinguish two cases according as $p$ is even or odd.

(a) $p=2 q$. Let $P$ and $Q$ be the functions defined by

$$
P(x)=1+\lambda\left(x^{q}+x^{-q}\right)+\mu\left(x^{q+1}+x^{-q-1}\right), \quad Q(x)=P(x)-\epsilon\left(x+x^{-1}\right),
$$

where $\epsilon$ is positive. Then

$$
Q^{m}(x)=P^{m}(x)+\sum_{k=0}^{(m-1)(q+1)+1} \eta_{m, k}(\epsilon)\left(x^{k}+x^{-k}\right)
$$

where

$$
\lim _{\epsilon \rightarrow 0} \eta_{m, k}(\epsilon)=0
$$

But it is easily shown that every integer $k$ satisfying $|k| \leqq q(q+1)$ can be written in the form $k=r q+s(q+1)$, where $r$ and $s$ are integers satisfying

$$
|r|+|s| \leqq q
$$

Therefore

$$
P^{q+j}(x)=\sum_{k=0}^{(q+j)(q+1)} \alpha_{q+j, k}\left(x^{k}+x^{-k}\right), \quad j=0,1, \cdots, q-1,
$$

and 


$$
\sum_{j=0}^{q} \frac{1}{j !} P^{j}(x)=\sum_{k=0}^{q(q+1)} \beta_{k}\left(x^{k}+x^{-k}\right),
$$

where $\alpha_{q+j, k}>0, \beta_{k}>0$. But (10), (11), (12) imply

$$
\exp [Q(x)]=\sum_{k=0}^{\infty} \gamma_{k}\left(x^{k}+x^{-k}\right)
$$

where $\gamma_{k} \geqq 0$ if $\epsilon$ is small enough. It follows that the function $g$ defined by

$$
g(t)=\exp \left[Q\left(e^{2 i t}\right)-Q(1)\right]
$$

is a characteristic function. Clearly $g$ divides $f$ and $g$ is not infinitely divisible. The theorem is proved in this case.

(b) $p=2 q-1$. We define now $P$ and $Q$ by

$$
\begin{aligned}
& P(x)=1+\lambda\left(x^{2 q-1}+x^{1-2 q}\right)+\mu\left(x^{2 q+1}+x^{-2 q-1}\right), \\
& Q(x)=P(x)-\epsilon\left(x+x^{-1}\right),
\end{aligned}
$$

where $\epsilon$ is positive. Then, using the fact that every integer $k$ satisfying $|k| \leqq 4 q^{2}$ can be written in the form $k=r(2 q-1)+s(2 q+1)$ where $r$ and $s$ are integers satisfying

$$
|r|+|s| \leqq 2 q,
$$

we obtain evident analogues of (10), (11) and (12) and therefore the validity of (13) if $\epsilon$ is small enough. It follows that the function $g$ defined by

$$
g(t)=\exp \left[Q\left(e^{i t}\right)-Q(1)\right]
$$

is a characteristic function and, since $g$ divides $f$ and is not infinitely divisible, the theorem is proved.

With the same method, we can prove that the following infinitely divisible characteristic functions do not belong to $I_{0}$ :

$$
\begin{aligned}
f_{1}(t)=\exp \left[\lambda_{1}\left(e^{i p t}-1\right)+\lambda_{2}\left(e^{i(p+1) t}-1\right)\right. \\
\left.+\lambda_{3}\left(e^{-i q t}-1\right)+\lambda_{4}\left(e^{-i(q+1) t}-1\right)\right],
\end{aligned}
$$

where $p$ and $q$ are integers greater than one and $\lambda_{j}$ are positive constants $(j=1,2,3,4)$;

(b) $f_{2}(t)=\exp \left[\mu_{1}\left(e^{i p t}-1\right)+\mu_{2}\left(e^{i(p+1) t}-1\right)+\mu_{3}\left(e^{-i t}-1\right)\right]$,

where $p$ is an integer greater than one and $\mu_{j}$ are positive constants $(j=1,2,3)$;

(c) $f_{3}(t)=\exp \left[\nu_{1}\left(e^{i p t}-1\right)+\nu_{2}\left(e^{i(p+1) t}-1\right)+\nu_{3}\left(e^{i t}-1\right)\right]$, 
where $p$ is an integer greater than two and $\nu_{j}$ are positive constants $(j=1,2,3)$.

Finally, we mention an open problem: Let $f$ be an infinitely divisible characteristic function such that $f_{N} f_{+}$and $f_{N} f_{-}$belong to $I_{0}$. If $f_{N}$ is nondegenerate, is it possible that $f$ does not belong to $I_{0}$ ?

\section{REFERENCES}

1. H. Cramér, On the factorization of certain probability distributions, Ark. Mat. 1 (1949), 61-65.

2. Yu. V. Linnik, Decomposition of probability distributions, Oliver and Boyd, Edinburgh, 1964.

3. E. Lukacs, Characteristic functions, Griffin, London, 1960.

4. I. V. Ostrovskir, On the decomposition of the infinitely divisible laws without Gaussian factor, Zap. Meh.-Mat. Fak. Har'kov. Gos. Univ. Har'kov. Mat. Obšc. 32 (1966), 51-72. (Russian)

5. R. Shimizu, On the decomposition of infinitely divisible characteristic functions with a continuous Poisson spectrum, Ann. Inst. Statist. Math. 16 (1964), 387-407.

The Catholic University of America and

Faculte des Sciences, Montpellier, France 